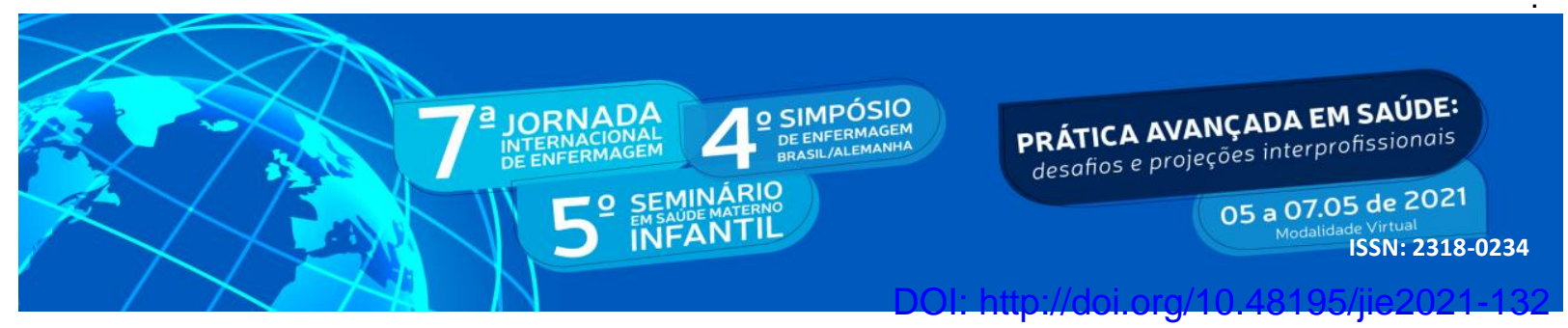

\title{
EXPERIÊNCIAS DE ESTUDANTES DE ENFERMAGEM NA CAMPANHA DE VACINAÇÃO CONTRA À COVID-19 EM SANTA MARIA/RS ${ }^{1}$
}

\author{
Laís Freitas Zuge ${ }^{2}$; Lorena Alves Fiorenza ${ }^{3}$; Giovana Luiza Rossato ${ }^{4}$ : Leandro da Silva \\ de Medeiros ${ }^{5}$; Maria Helena Gehlen ${ }^{6}$; Dirce Stein Backes ${ }^{7}$
}

\begin{abstract}
RESUMO
Objetivo: Descrever a experiência de estudantes de Enfermagem na campanha de vacinação contra à COVID-19. Método: Trata-se de um estudo descritivo, do tipo relato de experiência, desenvolvido por discentes de enfermagem da Universidade Franciscana no período de fevereiro a março de 2021. Resultados: A participação dos acadêmicos na Campanha de Vacinação contra à COVID-19 permitiu perceber a importância da enfermagem para a ocorrência das vacinações, participação na organização das atividades, vivenciar momentos de gratidão com os público vacinado e adquirir conhecimento prático em um contexto pandêmico. Conclusão: A experiência dos acadêmicos demonstrou-se de extrema importância na formação pessoal e profissional dos discentes inseridos, pois adquiriram e apropriaram habilidades e competências, bem como, o reconhecimento do enfermeiro como profissional essencial na organização e logística das vacinas.
\end{abstract}

Palavras-chave: Enfermagem; Estudantes de Ciências da Saúde; Infecções Por Coronavírus; Vacinação.

\begin{abstract}
Objective: To describe the experience of nursing students in the vaccination campaign against COVID-19. Method: This is a descriptive study, of the type of experience report, developed by nursing students from the Franciscan University from February to March 2021. Results: The participation of the students in the Vaccination Campaign against COVID-19 allowed us to perceive the importance of nursing for the occurrence of vaccinations, participation in the organization of activities, experience moments of gratitude with the vaccinated public and acquire practical knowledge in a pandemic context. Conclusion: The experience of the students proved extremely important in the personal and professional training of the inserted students, because they acquired and appropriated skills and competencies. As well as the recognition of nurses as an essential professional in the organization and logistics of vaccines.
\end{abstract}

\footnotetext{
${ }^{1}$ Relato de Experiência.

${ }^{2}$ Estudante do Curso de Enfermagem - Universidade Franciscana. E-mail: laiszugee@gmail.com

${ }^{3}$ Estudante do Curso de Enfermagem - Universidade Franciscana. E-mail: lorenafiorenzza@ gmail.com

${ }^{4}$ Estudante do Curso de Enfermagem - Universidade Franciscana. E-mail: rossatogiovana@ gmail.com

${ }^{5}$ Estudante do Curso de Enfermagem - Universidade Franciscana. E-mail: leandro.medeiros@ufn.edu.br

${ }^{6}$ Coorientadora. Doutora em Enfermagem. Docente do Curso de Enfermagem da Universidade Franciscana UFN. E-mail: Gehlenmh@gmail.com

${ }^{7}$ Orientadora. Doutora em Enfermagem. Docente do Curso de Enfermagem e Coordenadora do Mestrado

Profissional Saúde Materno Infantil da Universidade Franciscana. E-mail: backesdirce@ufn.edu.br
} 


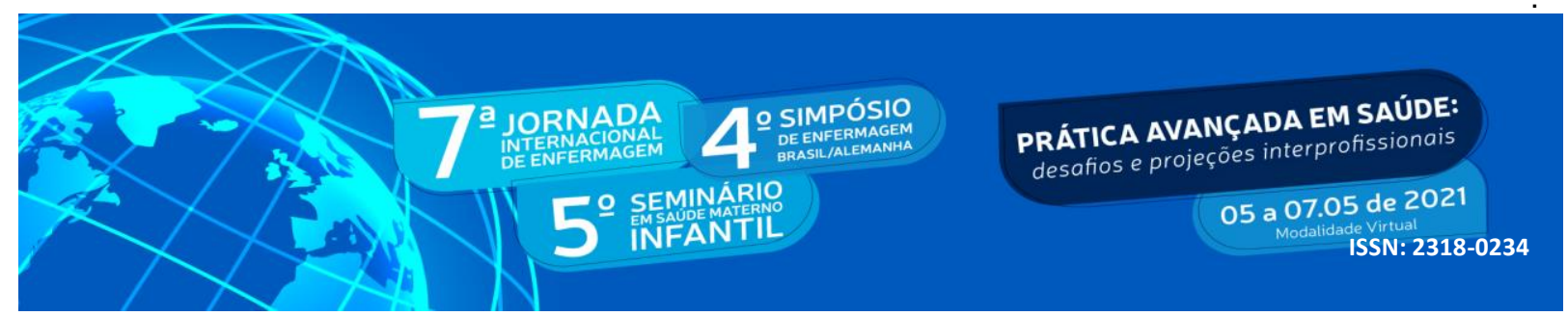

Key Words: Nursing; Students, Health Occupations; Coronavirus Infections; Vaccination.

\section{INTRODUÇÃO}

A pandemia da COVID-19 é causada por um coronavírus chamado de SARV-CoV-2. O coronavírus integra uma grande família de vírus que ocasionam doenças respiratórias em humanos e manifestam-se com resfriados comuns até patologias mais graves e raras. Os primeiros casos da COVID-19 foram relatados na cidade de Wuhan, na China, em dezembro de 2019. Desde então os casos aumentaram e espalharam-se, passando mais de 126.000.000 milhões de contaminados e 2.769.473 de óbitos até 28 de março de 2021, em nível mundial (WHO, 2021).

As medidas iniciais para combater a doença avassaladora, foram ações de prevenção para controlar o coronavírus. Dentre as medidas abrangeram desde lavagem constante das mãos com água e sabão ou uso de álcool em gel, proteção da boca com o antebraço quando tossir e/ou espirrar, manter distanciamento de pelo menos 1 metro das outras pessoas, uso de máscara e entre outras medidas de proteção e prevenção (ANS, 2020). Entretanto, a ferramenta mais eficaz contra o coronavírus, encontra-se no treinamento do sistema imunológico humano, adquirido por meio da vacina (WHO, 2021).

Por esse motivo, nos dias 11 e 12 de fevereiro de 2020, a OMS convocou um Fórum Global de Pesquisa e Inovação para debater diversos assuntos, sendo um deles, a Pesquisa e Desenvolvimento (P\&D) da vacina contra a COVID-19. A partir disso, foram lançadas sete vacinas em três plataformas, mas, outras 200 vacinas candidatas adicionais foram sendo desenvolvidas pelos cientistas de todo o mundo. Por conta disso, a OMS validou a primeira vacina para uso emergencial, no dia 31 de dezembro de 2020, sendo a imunizante da Pfizer/BioNTech (WHO, 2020, 2021).

No contexto brasileiro, o início da vacinação ocorreu em janeiro de 2021, quando o Ministério da Saúde publicou o Plano Nacional de Operacionalização da Vacinação contra a Covid-19. Até o momento da criação desse documento, a Agência Nacional de Vigilância Sanitária (ANVISA) autorizou o uso emergencial de dois tipos de vacina, sendo elas: AstraZeneca (Fiocruz) e Sinovac (Instituto Butantan) (BRASIL, 2021). 


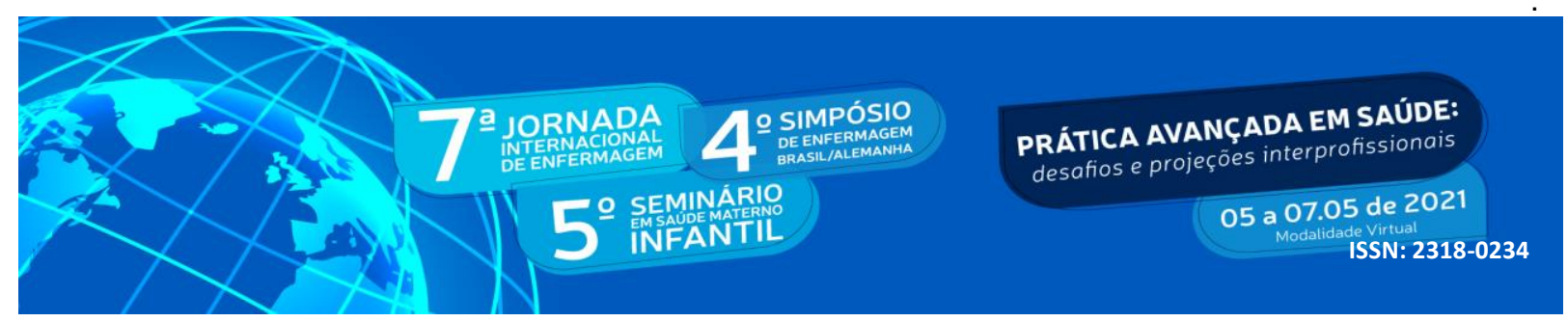

Diante disso, estabeleceu-se grupos prioritários para a vacinação, sendo eles: pessoas com 60 anos ou mais institucionalizadas ou não, pessoas com deficiência institucionalizadas, povos indígenas vivendo em terras indígenas, trabalhadores de saúde, povos e comunidades tradicionais ribeirinhas e quilombolas, pessoas com comorbidades, pessoas com deficiência permanente grave, pessoas em situação de rua, população privada de liberdade, 20 funcionários do sistema de privação de liberdade, trabalhadores da educação do ensino básico e do ensino superior, forças de segurança e salvamento, forças armadas, trabalhadores de transporte rodoviário, metroviário, ferroviário, aéreo, aquaviário, além de caminhoneiros, trabalhadores portuários e industriais (BRASIL, 2021).

Os profissionais da Enfermagem têm grande representatividade na aplicação das vacinas, atuando em posições essenciais para que a campanha de vacinação seja realizada. Em todos os espaços de vacinação, os enfermeiros e técnicos de enfermagem são encarregados de conduzir as atividades, organizando estratégias e escalas, aplicando as vacinas e servindo como influenciadores sobre a importância da imunização (CASTILHO, 2021).

De tal modo, entre os profissionais de enfermagem atuantes nas ações de vacinação, possuem os acadêmicos que também estão colaborando significativamente nessas atividades. Por isso, relatar experiências sob o olhar dos acadêmicos na vacinação contra a COVID-19 é fundamental devido o contexto pandêmico ser causador de muitas incertezas e inseguranças na população mundial.

\section{OBJETIVO}

Este estudo tem por objetivo descrever a experiência de estudantes de Enfermagem na campanha de vacinação contra à COVID-19.

\section{METODOLOGIA}

Trata-se de um estudo descritivo, do tipo relato de experiência, segundo Ferreira (2019) este é um texto que descreve precisamente uma dada experiência que possa contribuir de forma relevante para sua área de atuação. Determina-se pela descrição que um autor ou uma equipe 


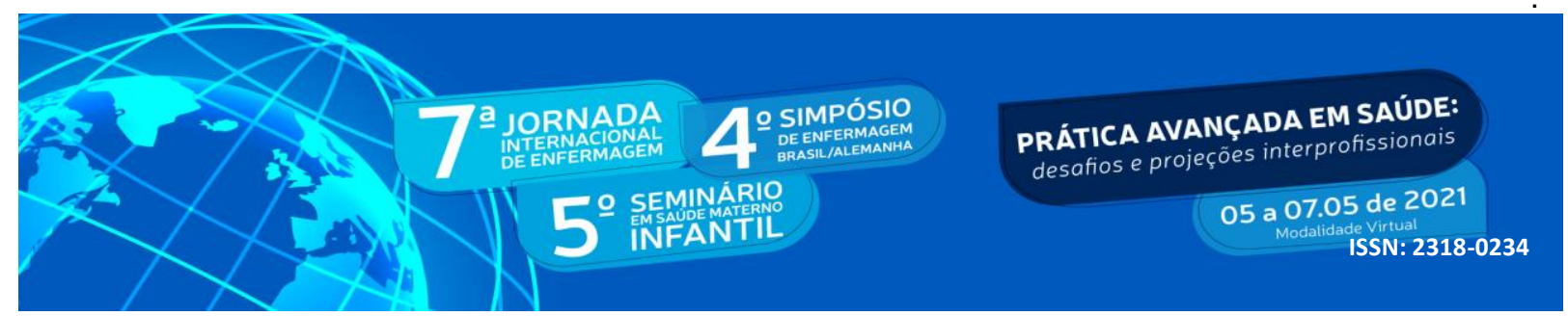

fazem de uma vivência profissional tida como exitosa.

O estudo foi desenvolvido por quatro discentes de enfermagem da Universidade Franciscana, localizada na região central do Rio Grande do Sul. O relato baseia-se na experiência dos estudantes quanto à participação na campanha de vacinação contra à COVID-19 do município de Santa Maria, Rio Grande do Sul.

O início da campanha de vacinação contra à COVID-19 deu-se em janeiro, organizada pela Prefeitura Municipal de Santa de Maria, Secretaria Municipal de Saúde e pelo Núcleo de Educação Permanente em Saúde (NEPeS). Ressalta-se que, a experiência em tela, aconteceu no período de fevereiro a março de 2021, sob supervisão de professores, a partir do vínculo institucional com o NEPeS de Santa Maria.

\section{RESULTADOS E DISCUSSÃO}

O ano de 2020 foi marcado por uma pandemia que registrou inúmeras mortes pela infecção viral do Sars-Cov-2, configurado como um tipo de coronavírus. Este é responsável por causar infecção no sistema respiratório, tornando-se necessário, em inúmeros casos, cuidados intensivos e o uso de ventilação mecânica. O primeiro caso de infecção ocorreu em dezembro de 2019, na China e, desde então, muitos países estabeleceram o isolamento físico como principal forma de evitar a contaminação (LANA et al., 2020).

O Brasil, desde o primeiro momento, teve uma postura negligente em relação à pandemia, tornando-se um dos países com o maior número de mortes registradas no mundo. Segundo o jornal The New York Times (2021), até o dia 26 de março de 2021, o Brasil registrou 303.462 mortes e mais de 12 milhões de casos da doença. Assim, a vacinação torna-se uma maneira de amenizar o número de casos graves, aliviando a superlotação das unidades de tratamento intensivo, bem como dos locais de urgência e emergência, evitando um colapso no sistema de saúde.

O Programa Nacional de Imunizações (PNI) foi instituído em 1973 proporcionando uma nova etapa no âmbito da prevenção nas Políticas de Saúde Pública. Atualmente, o Brasil possui um dos melhores programas de vacinação do mundo e, além disso, torna-se acessível à toda população por fazer parte do Sistema Único de Saúde.

O PNI conta com uma parceria com o Instituto Butantan (IB) que, a partir da criação do o Programa de Autossuficiência Nacional em Imunobiológicos, em 1985, conseguiu 


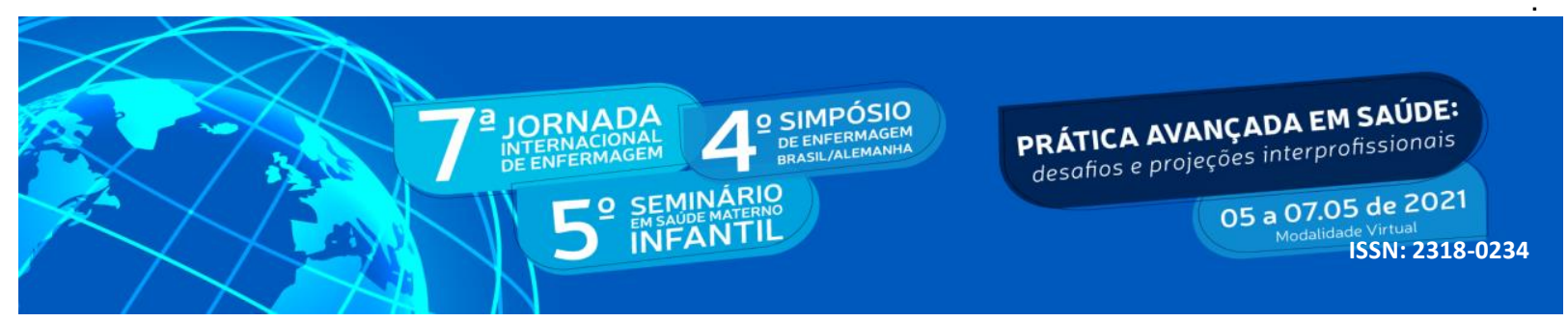

desenvolver um Centro de Biotecnologia para a produção de imunobiológicos (vacinas e soros) que, hoje, possuem o objetivo de atender as demandas da saúde pública brasileira (BRASIL, 2013).

A cidade de Santa Maria, localizada na região central do estado do Rio Grande do Sul, deu início às vacinações em 19 de janeiro de 2021 com os profissionais de saúde. Depois foram vacinados, respectivamente, idosos acamados e institucionalizados, idosos acima de 85 anos, profissionais liberais de saúde, idosos acima de 80 anos, idosos com 77 anos ou mais, idosos com 74 anos ou mais e idosos com 70 anos ou mais. Até o dia 19 de março, o município estava com cerca de 30.176 doses aplicadas, sendo 22.473 correspondentes à primeira dose e 7.703 correspondentes à segunda dose (PREFEITURA MUNICIPAL DE SANTA MARIA, 2021).

Figura 1: Relação das vacinas aplicadas no município de Santa Maria - RS

\begin{tabular}{|lrr|}
\hline Grupos Prioritários & $\mathbf{1}^{\mathbf{a}}$ Dose & $\mathbf{2}^{\mathbf{a}}$ Dose \\
\hline Trabalhadores da saúde & 10.740 & 3.269 \\
\hline 80 anos ou mais & 5.694 & 0 \\
\hline ILPIs & 653 & 455 \\
\hline Acamados & 561 & 0 \\
\hline Cuidadores & 552 & 0 \\
\hline Indígenas & 65 & 59 \\
\hline
\end{tabular}

Fonte: Prefeitura Municipal de Santa Maria, $2021^{8}$

Nessa questão, o trabalho voluntário nas campanhas de imunização do município são essenciais para o sucesso destas. A equipe que atua nessas ações é composta em sua grande maioria por acadêmicos de enfermagem das instituições de ensino superior presentes na cidade. Os estudantes participam de uma capacitação online e ficam aptos a atuar no cadastramento dos vacinados, na aplicação da vacina e na aspiração dos imunizantes.

A organização da vacinação acontece até um dia antes pela população, onde são formadas filas de carros em drive-thru, fazendo filas nas madrugadas para garantir as doses destinadas

\footnotetext{
${ }^{8}$ Disponível em: http://www.santamaria.rs.gov.br/vacinacao/?secao=vacinometro. Acesso em: 26 de março de 2021.
} 


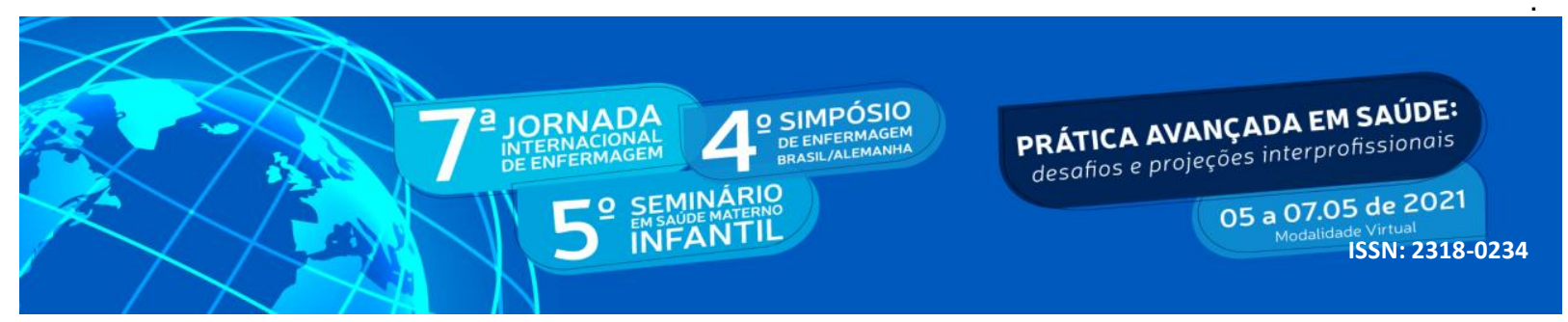

aos locais de aplicação. As vacinações acontecem em várias áreas da cidade de Santa Maria. A cidade de Santa Maria também conta com o colégio Marista para a campanha de vacinação, sendo assim o local mais central da cidade, porém sem o drive-thru. A organização dos locais de vacinação, até a aplicação da vacina se dá pela supervisão de professores, a partir de um vínculo institucional com o NEPeS de Santa Maria.

$\mathrm{Na}$ área de apoio onde os idosos passam para entregar seus documentos e serem redirecionados para a vacinação, nota-se a extrema felicidade, emoção, alguns chegam até a chorar pelo momento da vacinação contra a COVID 19 ter chegado a eles. Algumas pessoas presenteiam os vacinadores como forma de gratidão pelo trabalho realizado e felicidade por estar recebendo a vacina contra a COVID-19.

O apoio significa acolher o paciente para que ele sinta-se seguro para ir até a área da vacinação, todo amor depositado é no começo de tudo, onde a troca de olhares e palavras significam tanto para uma pessoa feliz que na maioria das vezes acabam chegando a noite para a fila da vacinação. Quando chega o seu momento, sua vez, o paciente está em êxtase de felicidade e uma emoção que às vezes nem consegue ser demonstrada.

\section{CONCLUSÃO}

Constata-se que, na vivência dos estudantes de enfermagem na campanha de vacinação aplicou-se o olhar de gestão e liderança frente às ações de imunizações. A experiência demonstra-se de extrema importância na formação pessoal e profissional dos discentes inseridos, pois adquiriram e apropriaram habilidades e competências.

Ressalta-se, que o profissional enfermeiro é extremamente importante na organização e logística das vacinas. Portanto, é válido reforçar a presença dos estudantes de enfermagem nas campanhas, para que vivenciam desde a graduação todo o processo necessário para a ocorrência do plano de vacinação, em tempos de pandemia. Bem como, são pessoas que colaboram para que as ações de vacinação sejam efetuadas e ganhem grandes avanços.

\section{REFERÊNCIAS}

ANS. Agência Nacional de Saúde Complementar. Prevenção é a principal medida para o combate à Covid-19, 2020. Disponível em: http://www.ans.gov.br/aans/noticias- 


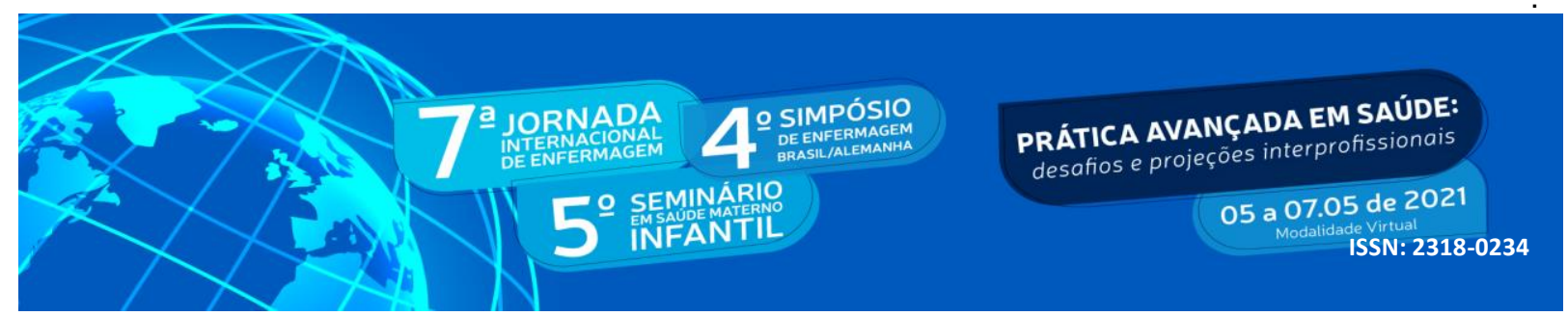

ans/coronavirus-covid-19/coronavirus-todas-as-noticias/6085-prevencao-e-a-principalmedida-para-o-combate-a-covid-19. Acesso em: 30 mar. 2021.

BRASIL. Ministério da Saúde. Departamento de Imunização e Doenças Transmissíveis. Coordenação-Geral do Programa Nacional de Imunizações. Plano Nacional de Operacionalização da Vacinação contra a Covid-19. 2 ed. Brasília: Ministério da Saúde, 2021. Disponível

em: http://www.saude.pi.gov.br/uploads/warning_document/file/641/Plano_Nacional_de_Vacinaç ão Covid19.pdf. Acesso em 26 mar. 2021.

BRASIL. Ministério da Saúde. Secretaria de Vigilância em Saúde. Departamento de Vigilância Epidemiológica. Programa Nacional de Imunizações (PNI): 40 anos. Brasília: Ministério da Saúde, 2013. 236 p. : il. Disponível em: https://bvsms.saude.gov.br/bvs/publicacoes/programa_nacional_imunizacoes_pni40.pdf.

Acesso em 26 mar. 2021.

BRAZIL Coronavirus Map and Case Count. The New York Times, 26 de março de 2021. Disponível em: https://www.nytimes.com/interactive/2020/world/americas/brazilcoronavirus-cases.html. Acesso em: 26 mar. 2021.

CASTILHO, L. A Enfermagem como foco principal ao sucesso da vacinação contra a COVID-19. Nursing (São Paulo), [S. 1.], v. 24, n. 274, p. 5344-5345, 2021. Disponível em: http://revistas.mpmcomunicacao.com.br/index.php/revistanursing/article/view/1319. Acesso em: 30 mar. 2021 .

FERREIRA, E. C. Relatos de Experiência. Projeto de Pesquisa de conclusão de Especialização em Atenção ao Paciente Crítico: Urgência, Emergência e UTI (UNINTER). 2019.

LANA, R. M. et al. Emergência do novo coronavírus (SARS-CoV-2) e o papel de uma vigilância nacional em saúde oportuna e efetiva. Cad. Saúde Pública, Rio de Janeiro, v. 36, n. 3, e00019620, 2020. Disponível em: https://www.scielo.br/scielo.php?script=sci_arttext\&pid=S0102-311X2020000300301. Acesso em: 26 mar. 2021.

SANTA MARIA, Prefeitura Municipal de. Secretária de Saúde. Monitoramento da imunização Covid-19. 2021. Disponível em: http://www.santamaria.rs.gov.br/vacinacao/?secao=vacinometro. Acesso em: 26 mar. 2021.

WHO. World Health Organization. Coronavirus. Geneva: WHO, 2021. Disponível em:https://www.who.int/health-topics/coronavirus\#tab=tab_1. Acesso em: 30 mar. 2021 


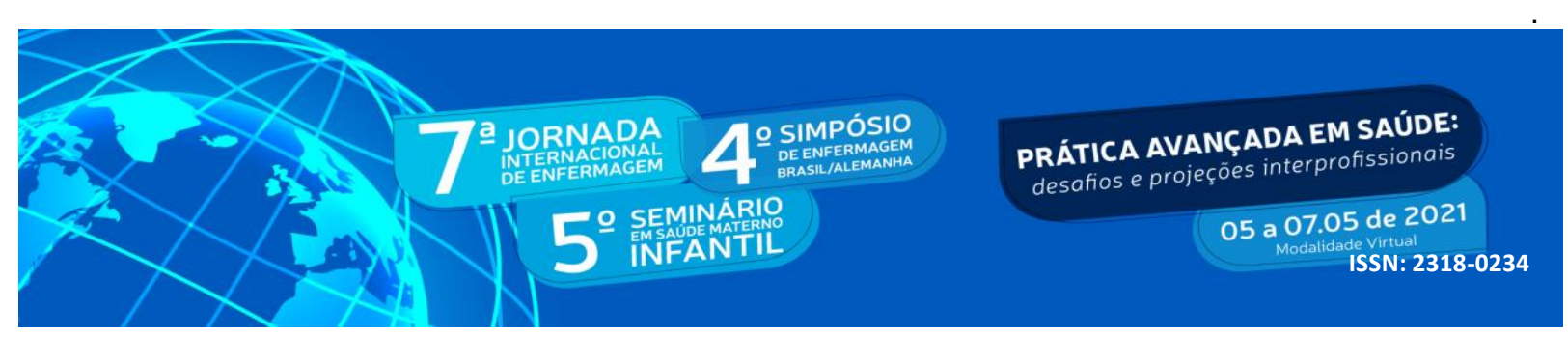

WHO. World Health Organization. COVID-19 Vaccines. Geneva: WHO, 2021. Disponível em: https://www.who.int/emergencies/diseases/novel-coronavirus-2019/covid-19-vaccines. Acesso em: 30 mar. 2021.

WHO. World Health Organization. R\&D Blueprint and COVID-19. Geneva: WHO, 2020. Disponível em: https://www.who.int/teams/blueprint/covid-19. Acesso em: 30 mar. 2021. 